\title{
A Low-Cost Solution for Automatic Plastic Segregation
}

\author{
Meher Madhu Dharmana, Aiswarya M. S.
}

\begin{abstract}
Solid waste management is a universal issue that matters to every single person in the world. The solid waste management system is fundamentally labor intensive with very little collection efficiency. The available automatic plastic segregation techniques are based on thermal imaging and electrostatic properties of materials- these methods are expensive for governments to invest upon, and also to maintain in landfills. In this paper, artificial intelligence techniques are exploited to recognize the sounds of plastics from that of other materials by designing suitable mechanism to produce sound from debris during segregation, the segregation process can be automated with relatively low-cost electronics like System on Chips and audio sensors. With 30,000 recorded samples of noisy plastic and non-plastic material sounds, ANN is trained and was able to successfully detect plastics with $93.5 \%$ accuracy in real time. Algorithms were developed in python and real time testing was done on SoC with a mic, which affirms that the proposed method is cost effective when compared to techniques involving image processing, thermal imaging and near infrared spectroscopy.
\end{abstract}

Keywords: Machine learning, features, classifier, audio signal.

\section{INTRODUCTION}

Urbanization creates problems in management of solid wastes such as health issues and environmental concern mainly in the urban areas of many countries. According to the Central Pollution Board (2015), India generated 1,43,449 of municipal solid waste per day during 2014-15, with an average waste generation of $0.11 \mathrm{~kg} / \mathrm{capita} /$ day (GIZ 2015) out of which plastic, paper and glass constitute 17 per cent [1]. Plastic waste generated by the industries as well as by the residents require urgent disposal as well as recycling of waste to avoid environmental and health issues. Plastic is responsible for contributing 16 percentage of chlorine in the environment and it also has fifty-four carcinogens. Highly toxic gases like phosgene, carbon monoxide, chlorine, Sulphur dioxide, nitrogen oxide, besides deadly dioxin is released when polythene bags are burnt irresponsibly. [2] In the absence of modernization and automation of waste management services, management od solid waste becomes

Revised Manuscript Received on February 05, 2020.

* Correspondence Author

Meher Madhu Dharmana*, Department of Electrical and Electronics Engineering, Amrita Vishwa Vidyapeetham, Amritapuri, India. E-mail: mehermadhud@am.amrita.edu

Aiswarya M S, Department of Electrical and Electronics Engineering, Amrita Vishwa Vidyapeetham, Amritapuri, India. E-mail: msaiswarya21@gmail.com

(C) The Authors. Published by Blue Eyes Intelligence Engineering and Sciences Publication (BEIESP). This is an open access article under the CC BY-NC-ND license (http://creativecommons.org/licenses/by-nc-nd/4.0/) labor- intensive task, also the collection efficiency appears to be less than 72 per cent in India. [3]

At present, there are few popular techniques to sort plastic and non-plastic materials. One of them is infrared hyper spectral imaging which is used for real-time instantaneous identification of plastics, where intelligent algorithms for image recognition and classification are used [6-11]. Near Infrared Spectroscopy is another technique used for the identification and selection of materials and is used for plastic segregation as proposed in [14]. Another common technique used in industries for material segregations is electrostatic separation as proposed in [12], but the major limitation of this process is that the materials should be made into granules and thus requires high infrastructural arrangements. All the existing sorting techniques encounter some snags like high capital and operation costs and are not economical for municipal solid waste management schemes. [16-18]

It is evident that the cost of material segregation is directly influenced by the technology used to detect the material. This paper shows a possible low-cost material detection technology which could significantly reduce the operating and installation costs of sorting systems. Material segregation in this paper is based on audio signal classification. Audio signals from different materials are transformed to data sets that represents the material explicitly using feature engineering. [4-7] There are many feature extraction techniques for audio signal [19] and these feature extraction methods use both spectral, and joint time-frequency signal representation. Artificial Neural Network is the classifier used to classify the feature database.

\section{PROPOSED METHOD}

The proposed mechanism employs audio signal analysis and machine learning to predict if a material is plastic or not. The low-cost plastic sensing and segregation mechanism is implemented using mic and System on Chip (SoC) using artificial intelligence algorithms. The basic block diagram of the algorithm is as shown in Figure.1.

The classification mechanism consists of the following steps, audio recording, audio feature extraction, the transformation of features, pre-processing of features, and lastly, classification. Plastic and metal are taken as testing materials for the database.

\section{A. Audio Recording}

Sound is generated on hitting and this sound is recorded at sampling frequency of $44.1 \mathrm{kHz}, 16$ bits/sample quantization and single channel. 


\section{B. Audio feature extraction}

In order to classify the recorded signal into plastic and metal, feature extraction is a critical step, as the database needs to be adequate to clearly distinguish the different classes of signals. Temporal analysis and spectral analysis are the two feature extraction techniques popularly used for audio signal analysis.

Here we use spectral analysis technique which uses spectral representation of audio signals for the analysis purpose. The most popularly used multidimensional feature vector in spectral analysis is Mel Frequency Cepstrum Coefficients (MFCC).

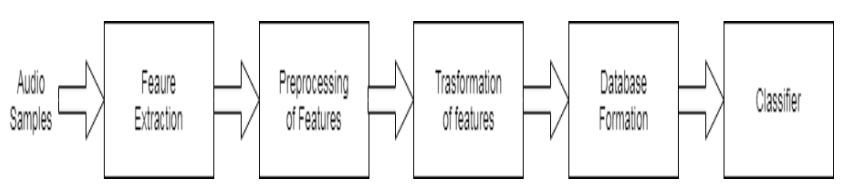

Figure 1. Block diagram of proposed algorithm

\section{B.1 Mel Frequency Cepstrum Coefficients}

Mel Frequency Cepstrum Coefficients (MFCC) is one among the dominant features used for audio analysis as it can represent a compact form of audio amplitude spectrum. The process of creating MFCC feature is described in Figure 2.

\section{B.2.3Bandwidth}

The average of the difference between spectral components of the signal and centroid can be referred to as bandwidth. It is given by,

$B(n)=\sqrt{\frac{\sum_{f=0}^{F_{m}}\left(f-f_{i}(n) \operatorname{TFD}(n, f)\right.}{\sum_{f=0}^{F_{m}} \operatorname{TFD}(n, f)}}$

\section{Preprocessing of features}

Data preprocessing is a data mining technique in which the real-world data which is often incomplete and inconsistent is transformed into understandable format. As a part of preprocessing, the following steps were carried out, checking for missing values, checking for categorical data, standardizing the data, Principle Component Analysis (PCA) in which it was found that all the 13 feature vectors obtained from MFCC are required and it is impossible to reduce the feature space and the last step data splitting.

\section{Classification - Artificial Neural Networks}

Brain inspired systems which are considered to copy the way humans learn are known as artificial neural networks.

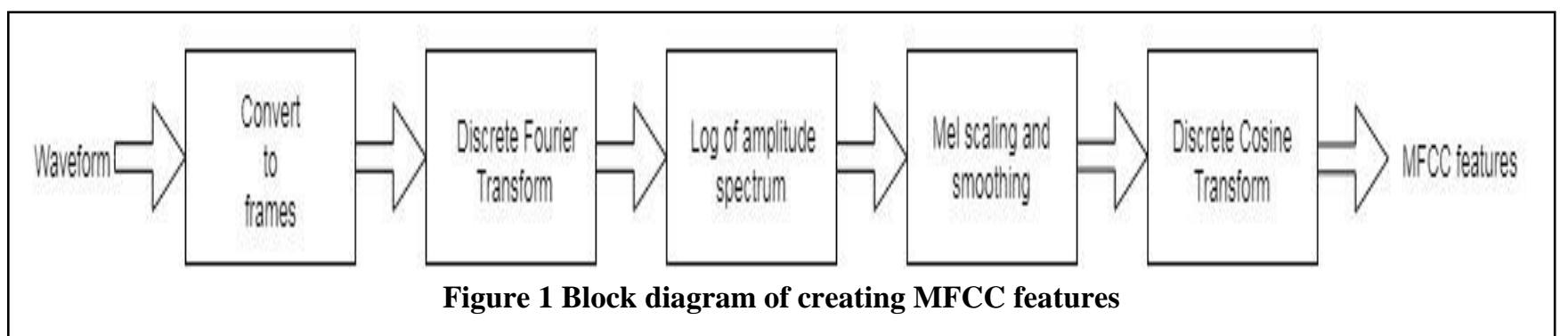

\section{B.2 Features extracted using Time Frequency Distribution Domain}

Features extracted from the two-dimensional time frequency domain (TFD) are listed below.

\section{B.2.1 Entropy}

Entropy property or feature of a signal is a spectral distribution measure and it describes the behavior of the signal like noise. In time frame $k$, entropy of a signal can be calculated as:

$$
\left.H(n)=\sum_{f=0}^{F m} P_{f}(T F D(n, f)) \log _{2} P_{f}(T F D)(n, f)\right)
$$

Where,

$$
P_{f}(\operatorname{TFD}(n, f))=\frac{T F D(n, f)}{\sum_{f=0}^{f=F_{m}} \operatorname{TFD}(n, f)}
$$

Here, $\operatorname{TFD}(\mathrm{n}, \mathrm{f})$ represents the energy of the signal at time frame $n$ and frequency index $f$ and $F_{m}$ refers to the maximum frequency.

\section{B.2.2 Brightness}

The brightness of a signal can be defined as the weighted midpoint of energy distribution in a given frame and is also referred to as the signal's frequency centroid. It is given by,

$$
f_{i}(n)=\frac{\sum_{f=0}^{F_{m}} f(T F D(n, f))}{\sum_{f=0}^{f_{m}}(T F D)(n, f)}
$$

This network consists of input as well as output layers, along with hidden layer which consist of units that are intended to transform the input in such a way that the output layer can use it. In the process of learning, a neural network finds the right function or the correct manner of transforming the input into the output. Here we use supervised learning in which the classification task depends upon labeled datasets.

\section{PLASTIC SEGREGATION}

The proposed low-cost technique uses sounds of metal and plastic recorded at $44.1 \mathrm{kHz}$ sampling frequency, 16 bits/sample quantization and single channel input by tapping the materials. Once the input data is recorded digital data is extracted and analyzed. Each audio signal is of 2 seconds long, mono-channel and these audio signals of metal and plastic are represented in Figure 3 and Figure 4 respectively. The classification process begins with the extraction of features, from each sound file and the features extracted using time frequency distribution of the metal and plastic are as shown in Figure 5 and Figure 6 respectively. 


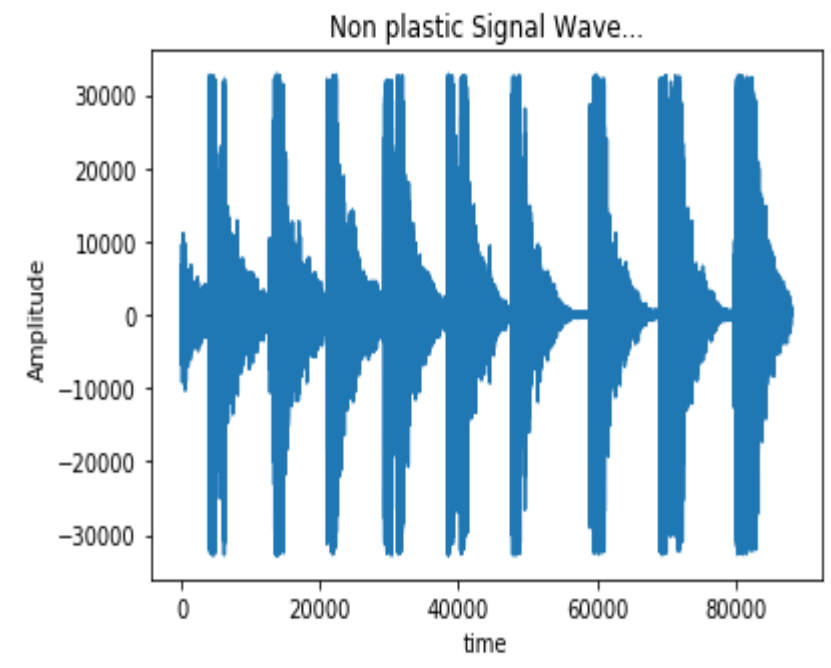

Figure 3. Audio signal of metal sound

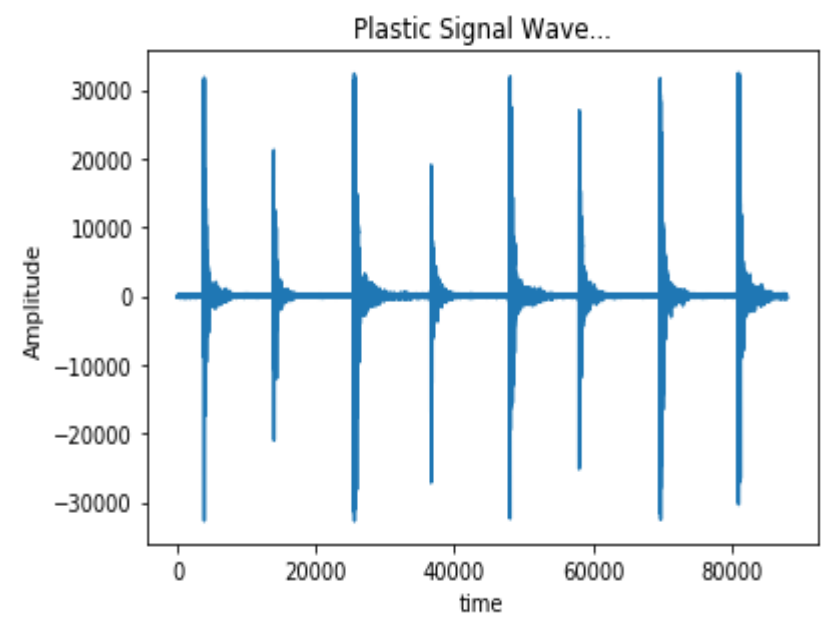

Figure 4. Audio signal of plastic sound

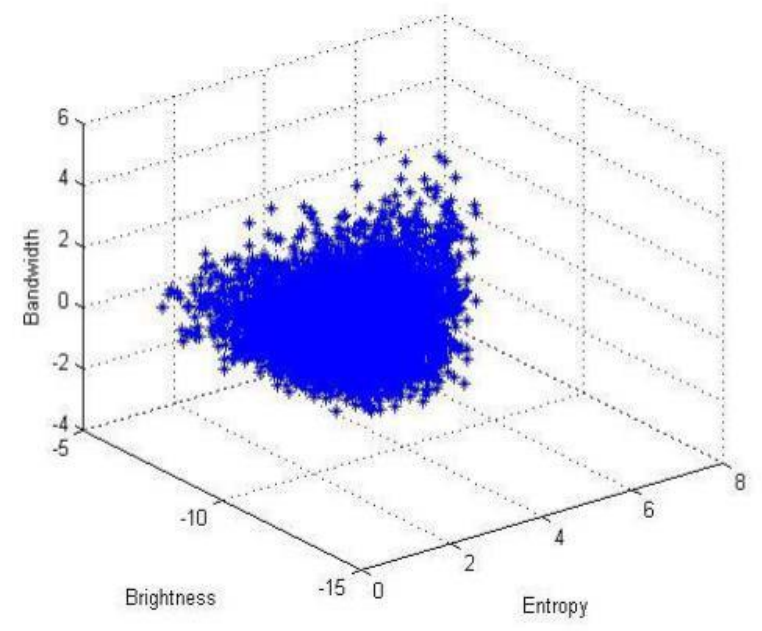

Figure 6. Extracted feature of plastic

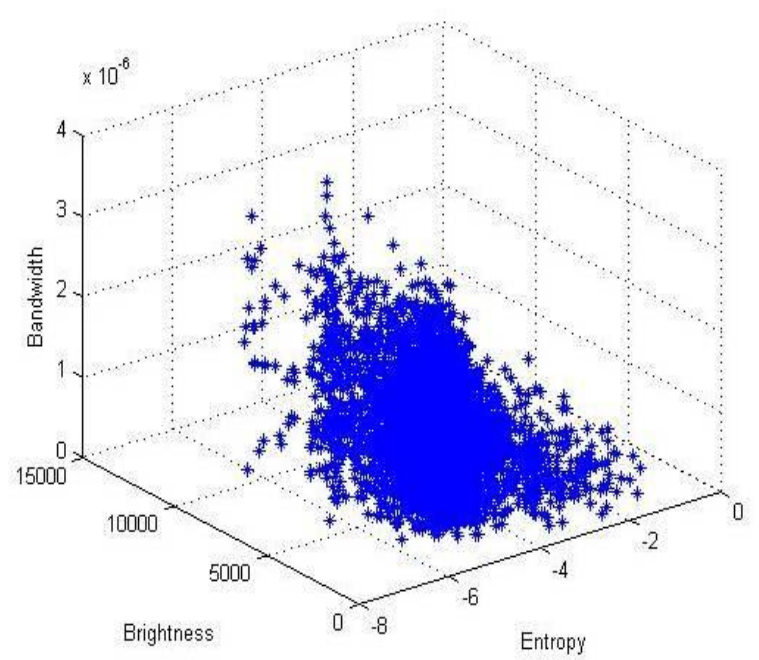

Figure 5. Extracted feature of metal

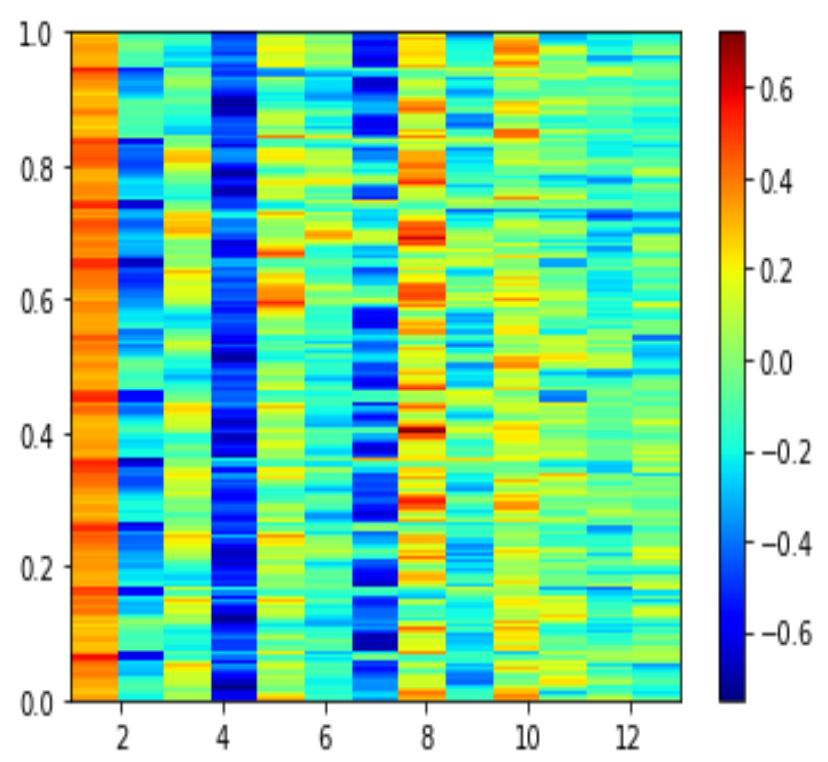

Figure 7. Plastic MFCC color map

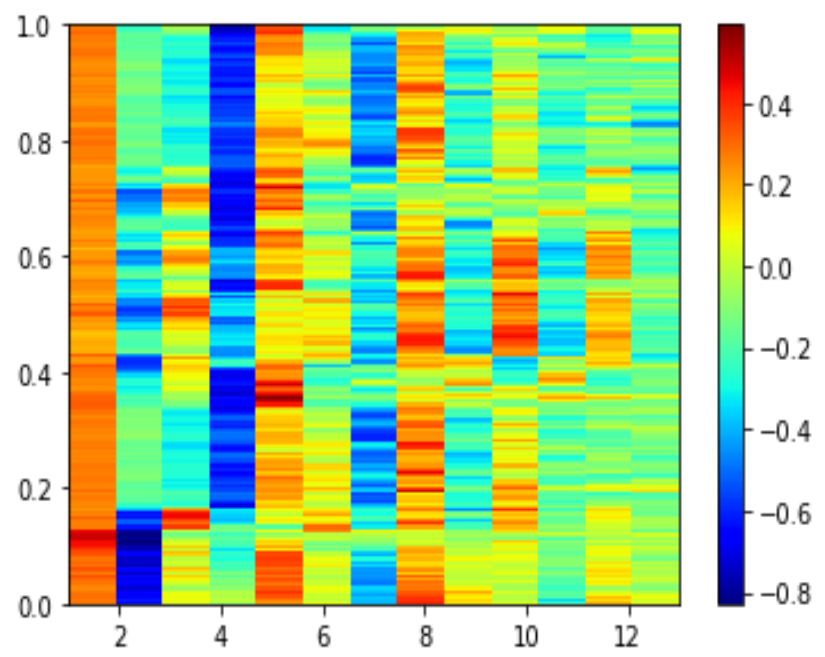

Figure 8. Metal MFCC color map

Published By:

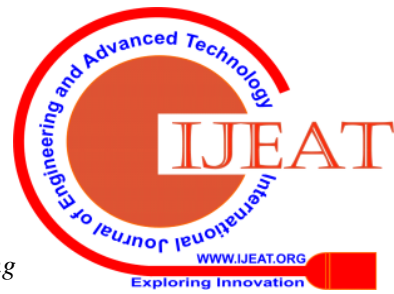


On giving the audio signal as input to MFCC Algorithm for coefficient extraction the unnecessary data is stripped away leaving behind the important information for classification. Even though higher order coefficients represent increasing levels of cepstral details, selecting large number of cepstral coefficients result in more complexity in the models. Thus 13 cepstral coefficients are selected after applying MFCC feature extraction process to the audio signal which are optimum for the analysis. On obtaining the MFCC features, the pre-processing steps are carried out. The pre-processed data is then fed to a neural network with 16 nodes in the input layer, 12 nodes in the hidden layer and 1 node in the output layer.

\section{RESULT AND ANALYSIS}

Figure 3 and Figure 4 represents the audio signals of metal and plastic sounds recorded at $44.1 \mathrm{kHz}$ sampling frequency. Features extracted from the audio signals - brightness, entropy, bandwidth and MFCC of plastic and metal are shown in the Figures 5-8. On feeding the above-mentioned features to the artificial neural network a mean accuracy of $93.5 \%$ and a variance of 0.015 were obtained. The confusion matrix of the classification is given in Table 1. Evaluation measures like error rate, sensitivity or recall, specificity, precision and false positive rate are listed in Table 2

Table 2. Confusion Matrix

\begin{tabular}{|c|c|c|}
\hline \multicolumn{3}{|c|}{ Confusion Matrix } \\
\hline & $\begin{array}{c}\text { Plastic } \\
\text { (Predicted) }\end{array}$ & Metal (Predicted) \\
\hline Plastic (Actual) & 132 & 3 \\
\hline Metal (Actual) & 8 & 107 \\
\hline
\end{tabular}

Table 2. Evaluation Measures

\begin{tabular}{|l|l|}
\hline Evaluation Measure & Calculated Value \\
\hline Error Rate & 0.044 \\
\hline Sensitivity or Recall & 0.97 \\
\hline Specificity & 0.93 \\
\hline Precision & 0.942 \\
\hline False Positive Rate & 0.069 \\
\hline
\end{tabular}

\section{CONCLUSION AND FUTURE SCOPE}

A low-cost plastic sensing and in turn segregating mechanism is proposed using mic and SoC taking advantage of artificial intelligence algorithms. Brightness, bandwidth, entropy and MFCC of the audio signals are selected as features for classification. The results proved that proper pre-processing of data could assist the classifier in detecting the plastic with $93.5 \%$ accuracy ideally. We kept normal ranges of performance metrics to compare and say the results are positive. The evaluation measures like sensitivity, specificity and precision have best value at 1 and from the results it is found as $0.97,0.93,0.942$ respectively and other measures like error rate and false positive rate have best value at 0 and from the results of the proposed mechanism it is found as 0.044 and 0.069 respectively. In future, the whole system can be re-designed in such a way that it works under any environmental conditions positively and can be implement in landfills.

\section{REFERENCES}

1. Shyamala Mani and Satpal Singh, "Sustainable municipal solid waste management in India: A Policy Agenda" Procedia Environmental Sciences, Elsevier, 2016.

2. Padgelwar, S., Nandan, A., \& Mishra, A. K. (2019). Plastic waste management and current scenario in india: A review. International Journal of Environmental Analytical Chemistry, doi: 10.1080/030 673 19.2019.

1686496

3. Gopalakrishnan, P., \& Ramaguru, R. (2019). Blockchain based waste management. International Journal of Engineering and Advanced Technology, 8(5), 2632-2635

4. Dadula, C. P., \& Dadios, E. P. (2017). Fuzzy logic system for abnormal audio event detection using mel frequency cepstral coefficients. Journal of Advanced Computational Intelligence and Intelligent Informatics, 21(2), 205-210. doi:10.20965/jaciii.2017.p0205

5. Zhao, P., Bai, M., \& Yang, J. (2017). Train safety detection technology based on audio analysis. Paper presented at the ACM International Conference Proceeding Series, 399-403.doi:10.1145/3158233.315936 8 .

6. Sharma, G., Umapathy, K., \& Krishnan, S. (2020). Trends in audio signal feature extraction methods. Applied Acoustics, 158 doi: 10.1016/j.apacoust.2019.107020

7. Ahmad, F., \& Sahil. (2019). Music genre classification using spectral analysis techniques with hybrid convolution-recurrent neural network. International Journal of Innovative Technology and Exploring Engineering, 9(1), 149-154. doi:10.35940/ijitee. A3956.119119

8. Rafeeq, M., Ateequrahman, A., Alam, S., \& Mikdad, M. (2016). Automation of plastic, metal and glass waste materials segregation using arduino in scrap industry. Paper presented at the Proceedings of the International Conference on Communication and Electronics Systems, ICCES 2016, doi:10.1109/CESYS.2016.7889840

9. Sreelakshmi, K., Akarsh, S., Vinayakumar, R., \& Soman, K. P. (2019) Capsule neural networks and visualization for segregation of plastic and non-plastic wastes. Paper presented at the 2019 5th International Conference on Advanced Computing and Communication Systems, ICACCS 2019, 631-636. doi:10.1109/ICACCS.2019.8728405

10. Shahbudin S, Hussain A, Wahab DA, Marzuki MM, Ramli S "Support vector machines for automated classification of plastic bottles.” In: 6th International Colloquium on Signal Processing and Its Applications (CSPA) (2010).

11. Witaya Srigul, Mongkol Kupimai, Prajuab Inrawong, "Plastic classification base on correlation of RGB color", 2016 13th International Conference on Electrical Engineering/Electronics, Computer, Telecommunications and Information Technology (ECTI-CON), DOI: 10.1109/ECTICon.2016.7561304.

12. Sejera, M., Ibarra, J. B., Canare, A. S., Escano, L., Mapanoo, D. C., \& Suaviso, J. P. (2017). Standalone frequency based automated trash bin and segregator of plastic bottles and tin cans. Paper presented at the IEEE Region 10 Annual International Conference, Proceedings/TENCON,2370-2372.doi:10.1109/TENCON.2016.78484 54

13. Tarun, K., Sreelakshmi, K., \& Peeyush, K. P. (2019). Segregation of plastic and non-plastic waste using convolutional neural network. Paper presented at the IOP Conference Series: Materials Science and Engineering.

14. Mary Cesetti, Leonardo Placentino, Giuseppe Pinese, Michele Libralato and PiergiorgioNicolosi, "Near infrared technology for material identification and selection" Published in: 2015 Fotonica AEIT Italian Conference on Photonics Technologies, DOI: 10.1049/cp.2015.0158. 
15. Thami Zeghloul Lucian Dascalescu, Lucian Dascalescu, Seddik Touhami, Mohamed Miloudi, Omar Dahou, Gontran Richard., Corona-assisted plate-type electrostatic separation process for granular plastic wastes, Published in: 2015 IEEE Industry Applications Society Annual Meeting, DOI: 10.1109/IAS.2015.7356772.

16. Nandhini, S., Mrinal, S. S., Balachandran, N., Suryanarayana, K., \& Ram, D. S. H. (2019). Electronically assisted automatic waste segregation. Paper presented at the Proceedings of the International Conference on Trends in Electronics and Informatics, ICOEI 2019, 846-850. doi:10.1109/ICOEI.2019.8862666

17. Gopinath, H., Indu, V., \& Dharmana, M. M. (2018). "Development of autonomous underwater inspection robot under disturbances". Paper presented at the Proceedings of 2017 IEEE International Conference on Technological Advancements in Power and Energy: Exploring Energy Solutions for an Intelligent Power Grid, TAP Energy 2017, 1-5. doi:10.1109/TAPENERGY.2017.8397219

18. Shamin, N., Mohamed Fathimal, P., Raghavendran, R., \& Prakash, K. (2019). Smart garbage segregation management system using internet of things (IoT) machine learning (ML). Paper presented at the Proceedings of 1st International Conference on Innovations in Information and Communication Technology, ICIICT 2019, doi: 10.1109 /ICIICT 1.2019.8741443

19. Huang, C. -., \& Lin, X. -. (2018). Study on machine learning based intelligent defect detection system. Paper presented at the MATEC Web of Conferences, 201 doi:10.1051/matecconf/201820101010

20. Dhiraj, Biswas, R., \& Ghattamaraju, N. (2018). An effective analysis of deep learning-based approaches for audio-based feature extraction and its visualization. Multimedia Tools and Applications, doi:10.100 7/s1 1042-018-6706-X

\section{AUTHORS PROFILE}

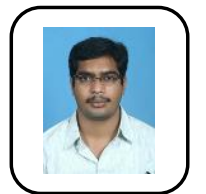

Mr. Meher Madhu Dharmana was born in Andra Pradesh, India on 1987. He received B.Tech. in Electrical Engineering from Jawaharlal Nehru Technological University, Kakinada, Andra Pradesh and M.Tech. in Instrumentation and Control systems from National Institute of Technology, Calicut, India. In 2012 he joined as Assistant Professor in Dept. of Electrical and Electronics Engineering, Amrita Vishwa Vidyapeetham, Kerala, India. His current research interests include Mechatronics and Artificial Intelligence. He has 12 Scopus indexed publications and one patent.

Ms. Aiswarya M S was born in Kerala, India on 1998. She is pursuing B.Tech. in Electrical and Electronics Engineering from Amrita Vishwa Vidyapeetham. Amritapuri, India. Her current research interests include Artificial Intelligence and machine learning. 